\title{
A Study to Find out Effect of Circuit Training on Balance in Stroke Patients- An Experimental Study
}

\author{
Dafda Renuka $\mathbf{H}^{1}$, Patel Prital $\mathbf{P}^{2}$, Sheikh Safa $\mathrm{A}^{3}$, Bhut Shivanee $\mathrm{S}^{4}$ \\ ${ }^{1}$ Assistant Professor, BN Patel College of Physiotherapy, N.S Patel Circle, Bhalej Road, Anand. \\ Gujarat -388001, India \\ 2,3,4 Intern, BN Patel College of Physiotherapy, N.S Patel Circle, Bhalej Road, Anand. Gujarat -388001, India
}

Corresponding Author: Dafda Renuka H

\begin{abstract}
Following stroke, patients lose functions of the motor, sensory and higher cognitive skills to various degrees which diminish their ability to balance effectively and make necessary postural adjustments. Circuit training was being used to improve balance in patients. Circuit training is a form of body conditioning that involves endurance training, resistance training, high intensity aerobic exercise and exercises performed in a circuit. Berg Balance Scale was used as an outcome measure.

Method: It is an experimental study done in 20 stroke patients selected with the help of purposive sampling method. Patients were selected on the basis of inclusion criteria and were divided into two groups. Group A received circuit training and Group B received conventional training. Training was given for 3 sessions per week for 4 weeks. Pre and Post BBS were taken.
\end{abstract}

Result: The study suggested that circuit training is more effective than conventional training in stroke patients.

Conclusions: The study shows that there is effect of circuit training on balance in stroke patient

Keywords: Stroke, Balance, Circuit training, BBS, Conventional training

\section{INTRODUCTION}

The term Stroke or brain attack is defined as the sudden loss of neurological function caused by an interruption of the blood flow to the brain. ${ }^{1}$ A stroke impairs balance of patient due to numbers of causes e.g. an injury to one of the systems that controls balance, damage to these systems or connections to the brain, injury to the brain, weakness of one side of body, loss of sensation, vision problems, dizziness, ataxia, side effects of drugs. ${ }^{1}$ Circuit training is a form of body conditioning that involves endurance training, resistance training, high intensity aerobic exercise and exercises performed in a circuit. Circuit training is composed of individual programs that focus on functional tasks. ${ }^{2}$ Thus, this study aims to find out effect of circuit training on balance in stroke patients using BBS.

\section{Need Of The Study}

There are already many protocols for circuit training but this protocol is easy and cost effective. It is less time consuming and also needs less space. It doesn't need advanced training. It doesn't require advanced equipments for the rehabilitation program. This protocol focuses on different types of activities. This can be used for generalized stroke patients. Circuit training is safe intervention and has no serious adverse effects. Circuit training which are commonly used are lengthy and it is tiring for patients, circuit training which is used in this study requires less time and frequency of session per week is also less. Circuit training can increase the length of each 
Dafda Renuka $H$ et.al. A study to find out effect of circuit training on balance in stroke patients- an experimental study.

activity to train the aerobic system. It targets on different components of balance. Hence, the aim of this study to find out effect of circuit training on balance in stroke patients using Berg Balance Scale.

\section{Aim Of The Study}

To find out effect of circuit training on balance in stroke patients using Berg Balance Scale.

\section{Objectives:}

To find out effect of circuit training on balance in stroke patients using Berg Balance Scale.

MATERIALS USED: Plinth, Chair, Stepup block, Slope, Cycle, Peg board, Spring, Stop watch, Data sheet, Assessment form, Consent form, Face mask, Sanitizer, Face shield, Gloves.

\section{CRITERIA FOR SELECTION: Inclusion Criteria:}

1. Age between 35 years to 60 years.

2. Gender: both male and female

3. Ischemic and hemorrhagic types of stroke.

4. Patients who are able to walk for at least 5 minutes.

5. Hemodynamically stable.

\section{Exclusion Criteria:}

1. Patients with history of any recent surgeries in low back and lower limbs.

2. Patients with history of any recent musculoskeletal injuries like fractures, dislocation, joint instability or any soft tissue injuries.

3. Patients with other form of neurological impairments

4. Blurred vision, vestibular system dysfunction

5. Patients with severe contractures due to spasticity.

6. Uncooperative patients or patient who is not willing to participate.

\section{METHOD}

The patient will be selected on the basis of inclusion and exclusion for the study purpose criteria was taken. Written informed consent was signed by each subject before proceeding for the study procedure. The demographic data including name, age, sex, affected side, post stroke duration, assistive devices for ambulation were recorded using subject evaluation form. Patients were then explained about the test and procedure to be conducted.

The study included one clinical test (Berg Balance Scale) to examine balance in people with stroke. For calculation of data in results, $\mathrm{p}$ value was set at $<0.05$. Before treatment Berg Balance Scale will be taken in all 20 patients. Patients are divided into two groups- Experimental Group and Control Group. Patients will be divided into Group A and Group B randomly. Group A will be Experimental group and Group B will be control group.

Circuit training will be given to Group A and Conventional training will be given to Group B.

\section{CIRCUIT TRAINING GROUP A:-}

- Stretching - hamstring stretch -Calf stretch -bicep stretch (30 second hold and 3 rep)

- Bridging

- Squatting

- Lunges

- Sit to stand

- Step-up and step-down

- Wall push-up

-Walking -5minutes

- Stationary Cycling -5 minutes

10 repetitions of each exercise and 2 set of each session.

40 to 45 minutes of each session $/ 3$ session per week /4 weeks session per month

CONVENTIONAL TRAINING GROUP B ;-

-stretching 
Dafda Renuka $H$ et.al. A study to find out effect of circuit training on balance in stroke patients- an experimental study.

-Active assisted movements of upper limb and lower limb

-Pelvic rotation

-Knee to chest

- One leg standing in parallel bar
-Hand exercise

10 repetitions of each exercise and 2 set of each session.

$\square 40$ to 45 minutes of each session $/ 3$ session per week /4week session per month

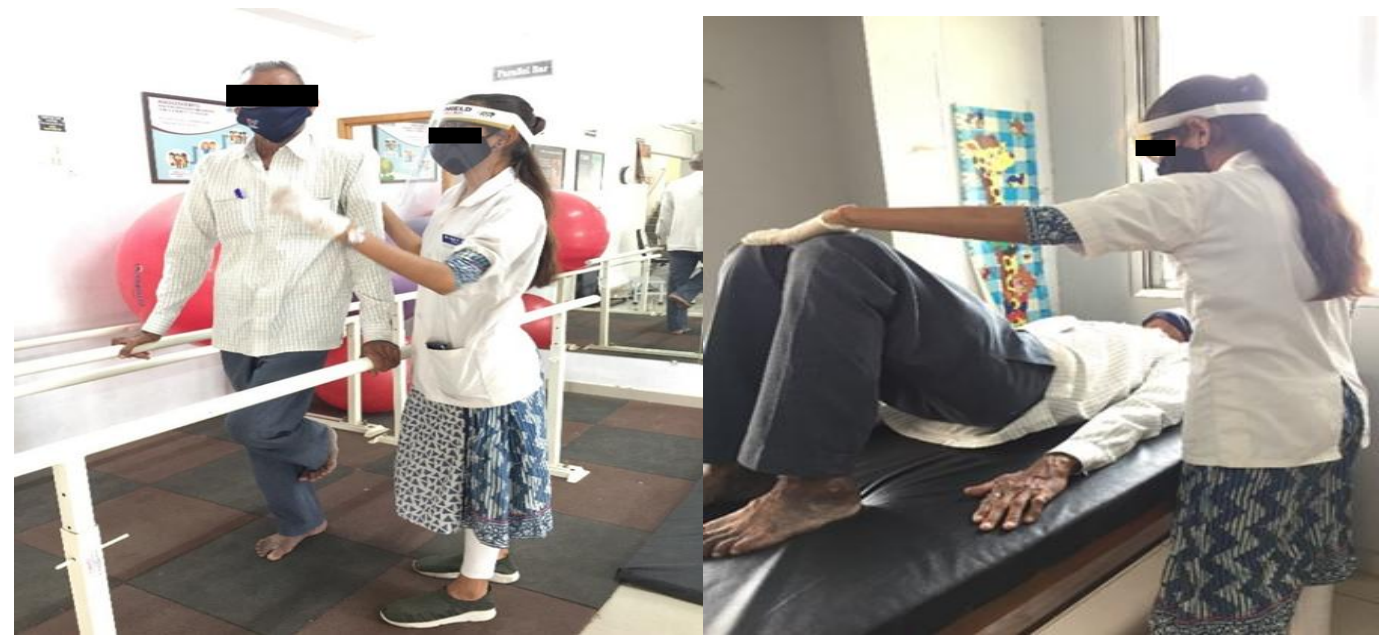

\section{RESULTS}

20 subjects were randomly divided into 2 groups. Group A received circuit training $(\mathrm{n}=10)$ and group received conventional therapy $(\mathrm{n}=10)$. Outcome measure Berg Balance Scale for balance was taken before and after completion of 12 sessions of the treatment. (3 sessions/week).

Paired ' $t$ ' test was used for pretreatment and post treatment comparison of Berg Balance Scale of Group A and Group B.

Table 1.1: Within group Comparison of Pre treatment and Post treatment Berg balance scale of Group A (paired 't' test) Paired samples statistics

\begin{tabular}{|ll|c|c|c|c|}
\hline & & $\mathbf{N}$ & Mean & Std. Deviation & Std. Error Mean \\
\hline Pair 1 & Pre test & 10 & 30.30 & 4.11 & 1.30 \\
\cline { 3 - 6 } & Post test & 10 & 37.70 & 3.77 & 1.19 \\
\hline
\end{tabular}

Paired differences

\begin{tabular}{|c|c|c|c|c|c|c|c|c|}
\hline & \multicolumn{5}{|c|}{ Paired differences } & \multirow[t]{3}{*}{$\mathrm{t}$} & \multirow{3}{*}{$\begin{array}{l}D \\
f\end{array}$} & \multirow{3}{*}{$\begin{array}{l}\text { Sig. (2- } \\
\text { tailed) }\end{array}$} \\
\hline & \multirow[t]{2}{*}{ Mean } & \multirow{2}{*}{$\begin{array}{l}\text { Std. } \\
\text { Deviation }\end{array}$} & \multirow{2}{*}{$\begin{array}{l}\text { Std. Error } \\
\text { Mean }\end{array}$} & \multicolumn{2}{|c|}{$95 \%$ confidence interval of the difference } & & & \\
\hline & & & & Lower & Upper & & & \\
\hline Pair 1 Pretest-Posttest & -7.40 & 1.77 & .56174 & -8.67075 & -6.12925 & -13.173 & 9 & .000 \\
\hline
\end{tabular}

\section{Mean Berg Balance Scale of Group A}

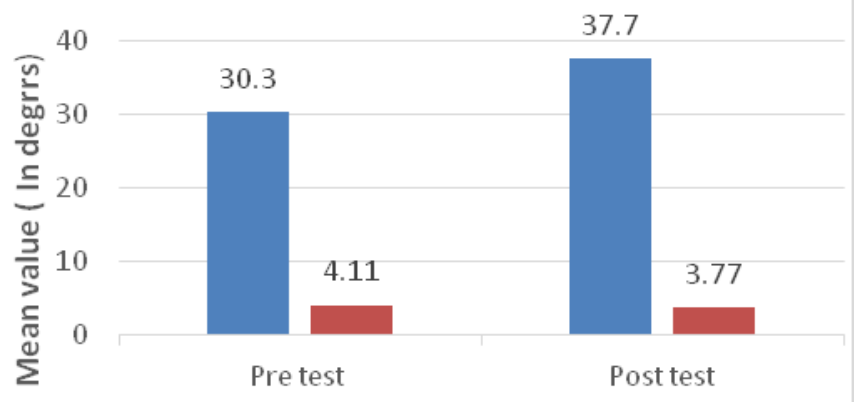

Mean Standard deviation

Graph 1.1 : Comparison of Pretreatment and Post treatment Berg balance scale of Group A (paired 't' test)

Interpretation: The above graph shows mean value of pre \& post Berg Balance Scale in Group A. 
Dafda Renuka $H$ et.al. A study to find out effect of circuit training on balance in stroke patients- an experimental study.

Table 1.2 : Comparison of pretreatment and post treatment Berg Balance Scale of group B ( paired ' $t$ ' test ) Paired samples statistics

\begin{tabular}{|ll|c|c|c|c|}
\hline & & $\mathrm{N}$ & Mean & Std. Deviation & Std. Error Mean \\
\hline Pair 1 & Pre test & 10 & 35.40 & 9.81 & 3.10 \\
\cline { 3 - 6 } & Post test & 10 & 44.60 & 9.99 & 3.15 \\
\hline
\end{tabular}

\begin{tabular}{|c|c|c|c|c|c|c|c|c|}
\hline \multicolumn{9}{|c|}{ Paired sample statics } \\
\hline & \multicolumn{5}{|c|}{ Paired differences } & \multirow[t]{3}{*}{$\mathbf{t}$} & \multirow[t]{3}{*}{ Df } & \multirow{3}{*}{$\begin{array}{l}\text { Sig. (2- } \\
\text { tailed) }\end{array}$} \\
\hline & \multirow[t]{2}{*}{ Mean } & \multirow{2}{*}{$\begin{array}{l}\text { Std. } \\
\text { Deviation }\end{array}$} & \multirow{2}{*}{$\begin{array}{l}\text { Std. Error } \\
\text { Mean }\end{array}$} & \multicolumn{2}{|c|}{$95 \%$ confidence interval of the difference } & & & \\
\hline & & & & Lower & Upper & & & \\
\hline Pair 1 Pretest-Posttest & -9.20 & 2.04 & .64 & -10.66216 & -7.73784 & -14.234 & 9 & .000 \\
\hline
\end{tabular}

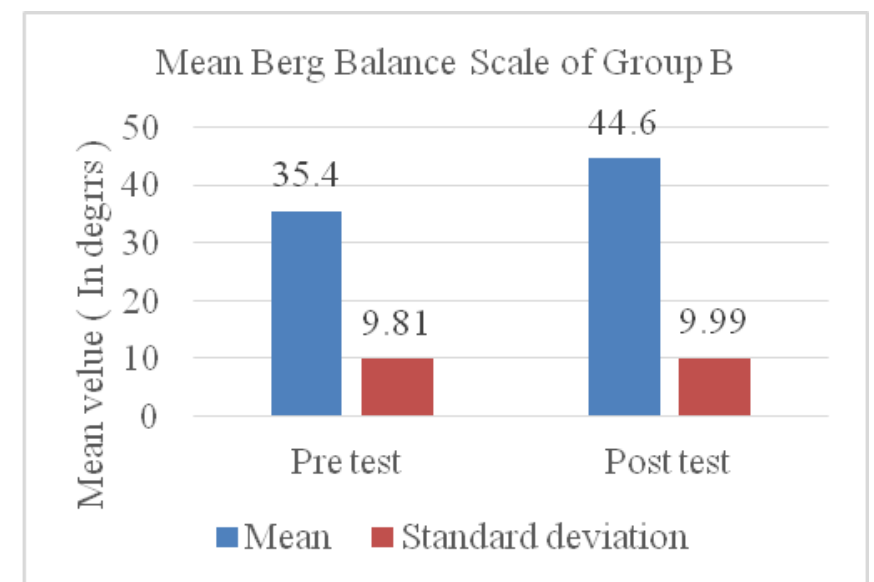

Graph 1.2:Comparison of pretreatment and post treatment mean of Group B

Interpretation: The above graph shows mean value of pre \& post Berg Balance Scale in Group B.

Table 1.3 : Comparison Between Group A and Group B -Berg Balance Scale(Independent t- test) Group Statistics

\begin{tabular}{|cc|c|c|c|c|}
\hline \multicolumn{2}{|c|}{ Treatment } & N & Mean & Std. Deviation & Std. Error Mean \\
\hline BBS & 1 & 10 & 44.60 & 9.99 & 3.15 \\
& 2 & 10 & 37.70 & 3.77 & 1.19 \\
\hline
\end{tabular}

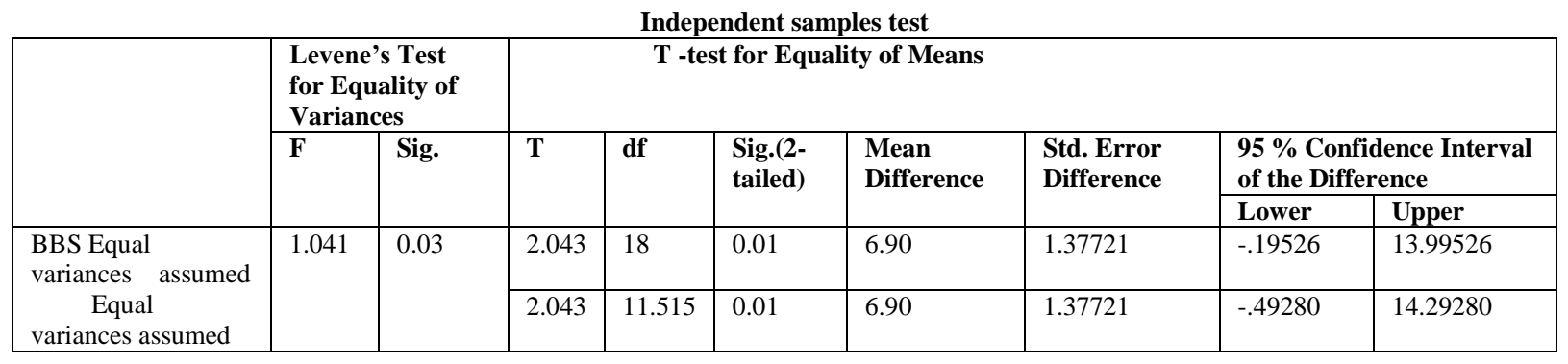

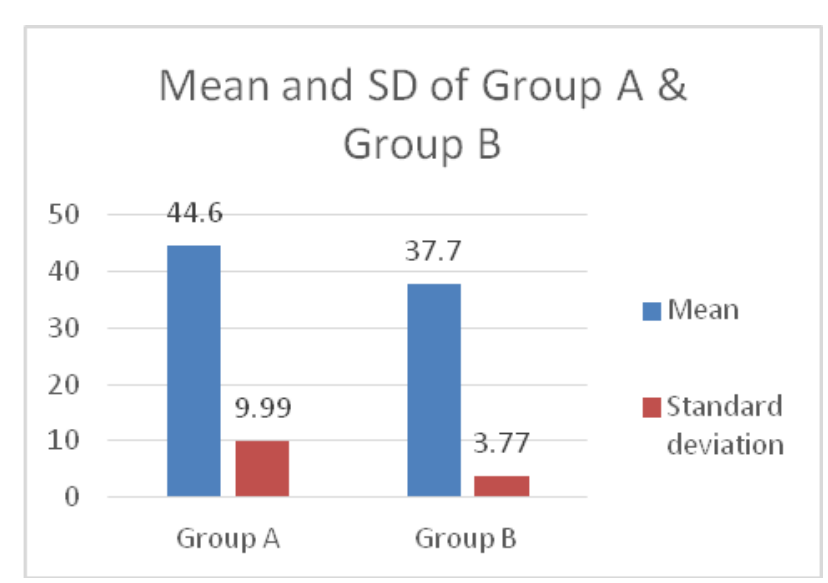

Graph 1.3: Comparison between group A and Group B 
Interpretation: The above graph shows Mean and SD value of Berg Balance Scale between Group A and Group B.

\section{DISCUSSION}

According to the result, study indicates that circuit training is more effective than conventional training to improve balance in stroke patients. The conventional training showed limited improvements in balance. It further shows improvement in reactive stepping measures and postural stability against stance perturbations. This training improved functional independence. It also helped in motor relearning.

Before treatment, Berg Balance Scale was taken in all 20 patients. Patients were divided into two groups- Experimental group and Control group. Patients were divided into Group A and Group B randomly. Group A- Experimental group and Group B- Control group. Circuit training was given to Group $\mathrm{A}$ and conventional training was given to Group B. Treatment was given for 4 weeks. The protocol of group A included the exercises which involved all the basic exercises which help in improving balance. The protocol of group B included the conventional exercises used in stroke rehab. 2 sets of 10 repetitions of each exercise were done. After 4 weeks post Berg Balance Scale was taken in all patients.

The circuit training focus on wholebody functional tasks translated into greater improvements in gait and the functionally based BBS.30 Circuit Class Therapy (CCT) is a form of Task Specific Training (TST) that involves the practice of structuring tasks in a circuit or series of workstations. ${ }^{3}$ The goal of CCT in stroke rehabilitation is to institute an enduring motor learning in order to optimize motor and functional recovery necessary for the achievement of community reintegration of stroke survivors. To accomplish sustained motor learning, rehabilitation must be geared towards a relatively permanent behavioral change, which is currently believed to manifest as a result of neuroplastic change in the brain itself. Compelling evidence from neuroscientific studies suggest that neuroplastic changes in the cerebral cortex and in other parts of the central nervous system (CNS) are the physiological mechanism for effective motor skill retraining following stroke. ${ }^{3}$ Clinically, in CCT, participants are exposed to multiple progressively structured tasks to be accomplished within a session, and considering the pathophysiological challenge of stroke survivors, they may need more time to perform multiple repetitions to enable neuroplastic changes. Sun Mi Kim et al.,(2015) concluded that circuit training therapy could be used as a cost-effective or alternative method to individual physiotherapy for improving lower-extremity, motor control, balance, walking competency and basic ADL. ${ }^{4}$

\section{Further Recommendation}

Larger sample size can be recommended for further study. Study can be performed to compare if any difference in recovery among acute, subacute and chronic patients.

\section{Limitations of the Study}

Patients with cognitive impairment were not included. Duration of stroke was not taken into consideration. Small sample size.

\section{CONCLUSION}

This study included customized circuit training protocol which showed positive effect on balance. This protocol is cost effective and requires less time. It is a simple protocol so patients can perform the activities easily. Thus, the circuit training is effective in improving balance in stroke patients.

\section{ACKNOWLEDGEMENT}

We would like to thank our family and our patients for support. 
Dafda Renuka $H$ et.al. A study to find out effect of circuit training on balance in stroke patients- an experimental study.

\section{Conflicts of Interest}

There was no personal or institutional conflict of interest for the study.

Source of Funding: No fund was needed.

Ethical Clearance: From BN Patel College Of Physiotherapy, Anand.

\section{REFERENCES}

1. Susan B O Sullivan, Thomas J Schmitz; Physical rehabilitation; 5th Edition; Jaypee Brothers; 2006.

2. World Health Organization. Preventing chronic Diseases: A vital investment. Geneva, Switzerland; 2005.
3. Lawal, I.U., Hillier, S.L., Hamzat, T.K. et al. Effectiveness of a structured circuit class therapy model in stroke rehabilitation: a protocol for a randomised controlled trial. BMC Neurol 15, $88 \quad$ (2015). https://doi.org/10.1186/s12883-015-0348-7

4. Sun Mi Kim et al. Clinical application of circuit training for subacute stroke patients: a preliminary study. J Phys Ther Sci. 2016 Jan;28(1):169-74. doi: 10.1589/jpts.28.169.

How to cite this article: Dafda Renuka H, Patel Prital P, Sheikh Safa A et.al. A study to find out effect of circuit training on balance in stroke patients- an experimental study. Int $J$ Health Sci Res. 2021; 11(7): 339-344. DOI: https://doi. org/10.52403/ijhsr.20210746 